\title{
Distance and fractional isomorphism in Steiner triple systems
}

\author{
A.D. Forbes, M.J. Grannell and T.S. Griggs \\ Department of Mathematics \\ The Open University \\ Walton Hall, Milton Keynes MK7 6AA \\ UNITED KINGDOM \\ tonyforbes@ltkz.demon.co.uk \\ m.j.grannell@open.ac.uk \\ t.s.griggs@open.ac.uk
}

\begin{abstract}
In [8], Quattrochi and Rinaldi introduced the idea of $n^{-1}$ - isomorphism between Steiner systems. In this paper we study this concept in the context of Steiner triple systems. The main result is that for any positive integer $N$, there exists $v_{0}(N)$ such that for all admissible $v \geq v_{0}(N)$ and for each $\operatorname{STS}(v)$ (say $S$ ), there exists an $\operatorname{STS}(v)$ (say $\left.S^{\prime}\right)$ such that for some $n>N, S$ is strictly $n^{-1}$-isomorphic to $S^{\prime}$. We also prove that for all admissible $v \geq 13$, there exist two $\operatorname{STS}(v) \mathrm{s}$ which are strictly $2^{-1}$-isomorphic.

Define the distance between two Steiner triple systems $S$ and $S^{\prime}$ of the same order to be the minimum volume of a trade $T$ which transforms $S$ into a system isomorphic to $S^{\prime}$. We determine the distance between any two Steiner triple systems of order 15 and, further, give a complete classification of strictly $2^{-1}$-isomorphic and $3^{-1}$-isomorphic pairs of STS(15)s.
\end{abstract}

This is a preprint of an article accepted for publication in Rendiconti del Circolo Matematico di Palermo. (C)2006 (copyright owner as specified in the journal).

AMS classification: 05B07

Keywords: Steiner triple system; configuration; trade; $n^{-1}$-isomorphism; fractional isomorphism. 


\section{Introduction}

A Steiner triple system of order $v$, briefly $\operatorname{STS}(v)$, is a pair $(V, \mathcal{B})$ where $V$ is a set of cardinality $v$ of elements, or points, and $\mathcal{B}$ is a collection of triples, also called blocks or lines, which has the property that every pair of distinct elements of $V$ occurs in precisely one triple. It is well known that an $\operatorname{STS}(v)$ exists if and only if $v \equiv 1$ or $3(\bmod 6)$. Such values of $v$ are called admissible. An $n$-line configuration is a collection of $n$ triples which has the property that every pair of distinct elements occurs in at most one triple. If $\mathcal{C}$ is a configuration, we denote the number of blocks by $b(\mathcal{C})$, the number of points by $p(\mathcal{C})$ and the set of points by $P(\mathcal{C})$. The degree of a point is the number of triples which contain it. Two configurations $\mathcal{C}$ and $\mathcal{D}$ are said to be isomorphic, $\mathcal{C} \cong \mathcal{D}$, if there exists a one-to-one mapping $\phi: P(\mathcal{C}) \rightarrow P(\mathcal{D})$ such that for each triple $T \in \mathcal{C}, \phi(T)$ is a triple in $\mathcal{D}$. Two $\operatorname{STS}(v) \mathrm{s},(V, \mathcal{B})$ and $\left(V^{\prime}, \mathcal{B}^{\prime}\right)$ are isomorphic if $\mathcal{B} \cong \mathcal{B}^{\prime}$. Up to isomorphism, the $\operatorname{STS}(3), \operatorname{STS}(7)$ and $\operatorname{STS}(9)$ are unique. There are two STS(13)s, 80 STS(15)s and, as shown recently by Kaski and Östergård [4], 11,084,874,829 STS(19)s. In this paper we confine our attention mainly to the 80 pairwise non-isomorphic STS(15)s, and we refer to them by the standard numbering as given in $[2$, Chapter 5].

A trade $T=\left\{\mathcal{T}_{1}, \mathcal{T}_{2}\right\}$ is a pair of disjoint $m$-line configurations $\mathcal{T}_{1}$ and $\mathcal{T}_{2}$ which has the property that every pair of distinct elements occurs in precisely the same number (zero or one) of triples of $\mathcal{T}_{1}$ as of $\mathcal{T}_{2}$. Traditionally, the number of lines, $m$, is called the volume of the trade, denoted by $\operatorname{vol}(T)$, and the foundation of the trade, found $(T)$, is the set of elements covered by $\mathcal{T}_{1}$ and $\mathcal{T}_{2}$. As it will be important to distinguish between a trade $T=\left\{\mathcal{T}_{1}, \mathcal{T}_{2}\right\}$ and either of the configurations $\mathcal{T}_{1}$ and $\mathcal{T}_{2}$, the latter will be referred to as tradeable configurations. If $S=(V, \mathcal{B})$ and $S^{\prime}=\left(V, \mathcal{B}^{\prime}\right)$ are two Steiner triple systems and $T=\{\mathcal{C}, \mathcal{D}\}$ is a pair of configurations with $\mathcal{C} \subseteq \mathcal{B}$ such that $S^{\prime}$ is isomorphic to $(V,(\mathcal{B} \backslash \mathcal{C}) \cup \mathcal{D})$, then we say that $T$ transforms $S$ into $S^{\prime}$. The trades $T=\left\{\mathcal{T}_{1}, \mathcal{T}_{2}\right\}$ and $T^{\prime}=\left\{\mathcal{T}_{1}^{\prime}, \mathcal{T}_{2}^{\prime}\right\}$ are said to be isomorphic if there exists a one-to-one mapping $\phi:$ found $(T) \rightarrow$ found $\left(T^{\prime}\right)$ such that $\phi\left(\left\{\mathcal{T}_{1}, \mathcal{T}_{2}\right\}\right)=\left\{\mathcal{T}_{1}^{\prime}, \mathcal{T}_{2}^{\prime}\right\}$. It is well-known that there exist trades of volume $n$ only for $n=4$ and $n \geq 6$ [6]; for example, there is a unique trade of volume 4, called a Pasch switch. A complete list of trades of up to 9 blocks is given in [3], from which it can be seen that every trade, $\left\{\mathcal{T}_{1}, \mathcal{T}_{2}\right\}$, of volume not exceeding 8 has $\mathcal{T}_{1} \cong \mathcal{T}_{2}$.

We will be interested in three basic questions. The first of these is as 
follows. Given two $\operatorname{STS}(v) \mathrm{s}, S$ and $S^{\prime}$, what is the minimum volume of a trade $T$ which transforms $S$ into (a system isomorphic to) $S^{\prime}$ ? Formally, we define this to be the distance, $d\left(S, S^{\prime}\right)$, between $S$ and $S^{\prime}$. Observe that $d\left(S, S^{\prime}\right)$ is a metric in the usual sense. We investigate the distance problem for STS(15)s and we note in passing that the distance between the cyclic STS(13) and the non-cyclic STS(13) is 4.

The other questions are motivated by a paper of Quattrocchi and Rinaldi [8], who introduce the concept of $n^{-1}$-isomorphism. Two configurations $\mathcal{C}$ and $\mathcal{D}$ are said to be $n^{-1}$-isomorphic if there are partitions $\mathcal{C}_{1}, \mathcal{C}_{2}, \ldots, \mathcal{C}_{n}$ of $\mathcal{C}$ and $\mathcal{D}_{1}, \mathcal{D}_{2}, \ldots, \mathcal{D}_{n}$ of $\mathcal{D}$ such that $\mathcal{C}_{i} \cong \mathcal{D}_{i}$ for $i=1,2, \ldots, n$. Two Steiner triple systems, $(V, \mathcal{B})$ and $\left(V^{\prime}, \mathcal{B}^{\prime}\right)$, are $n^{-1}$-isomorphic if $\mathcal{B}$ and $\mathcal{B}^{\prime}$ are $n^{-1}$-isomorphic. For $n \geq 2$, two configurations are said to be strictly $n^{-1}$ isomorphic if they are $n^{-1}$-isomorphic but not $(n-1)^{-1}$-isomorphic; similarly for Steiner triple systems. It is natural to call this concept, fractional isomorphism. (Note that this has a different meaning to that given in [9], in relation to graphs.) Clearly, $1^{-1}$-isomorphism is the same as isomorphism. However, unlike isomorphism, $n^{-1}$-isomorphism is not necessarily an equivalence relation if $n \geq 2$; reflexivity and symmetry are always satisfied but in general transitivity fails.

The second question is related to the first. If the trade $T=\left\{\mathcal{T}_{1}, \mathcal{T}_{2}\right\}$ transforms $S$ to $S^{\prime}$ and $\mathcal{T}_{1} \cong \mathcal{T}_{2}$, then $S$ and $S^{\prime}$ are $2^{-1}$-isomorphic. However, as noted in [3], there are trades consisting of non-isomorphic tradeable configurations. We ask the following question. For two non-isomorphic STS $(v) \mathrm{s}$, $S$ and $S^{\prime}$, what is the minimum volume of a trade $T$, consisting of isomorphic tradeable configurations, which transforms $S$ into (a system isomorphic to) $S^{\prime}$ ? Formally, we define this to be $h\left(S, S^{\prime}\right)$. If no such trade exists, we write $h\left(S, S^{\prime}\right)=\infty$. If $h\left(S, S^{\prime}\right)<\infty$, then $S$ and $S^{\prime}$ are $2^{-1}$-isomorphic. Although exceptions are relatively scarce, the converse is not necessarily true, as our investigations of the $h$ function for STS(15)s will reveal.

The third question is for what values of $n$ do there exist two $\operatorname{STS}(v) \mathrm{s}, S$ and $S^{\prime}$, which are strictly $n^{-1}$-isomorphic.

\section{Fractional isomorphism}

It was Kirkman [5] who gave the first proof that for all admissible $v$ there exists an $\operatorname{STS}(v)$. Later, Moore [7] proved that for all admissible $v \geq 13$, there exist two non-isomorphic $\operatorname{STS}(v)$ s; see [2, page 70]. Our next goal is to 
state and prove two existence theorems concerning $n^{-1}$-isomorphic $\operatorname{STS}(v) \mathrm{s}$.

Theorem 2.1 For all admissible $v \geq 13$, there exist two $S T S(v) s$ which are strictly $2^{-1}$-isomorphic.

We conjecture that for each positive integer $n$, there exists $v_{0}(n)$ such that for all admissible $v \geq v_{0}(n)$ there exist two $\operatorname{STS}(v)$ s which are strictly $n^{-1}$ isomorphic. Whilst we are unable to prove this conjecture, we can establish a weaker result in the same direction.

Theorem 2.2 For any positive integer $N$, there exists a positive integer $v_{0}(N)$ such that for all admissible $v \geq v_{0}(N)$ and for each $S T S(v)$ (say $S$ ), there exists an $S T S(v)$ (say $S^{\prime}$ ) such that for some $n>N, S$ is strictly $n^{-1}$-isomorphic to $S^{\prime}$.

Before dealing with these theorems we prove some lemmas.

Lemma 2.1 If $\mathcal{X}$ is a configuration, let $\rho(\mathcal{X})$ denote the (possibly empty) set of blocks obtained by removing from $\mathcal{X}$ all blocks containing points of degree 1. Suppose $\mathcal{C}$ and $\mathcal{D}$ are configurations which cover the same pairs, and suppose also that $\mathcal{C} \cong \mathcal{D}$. Then $\rho(\mathcal{C}) \cong \rho(\mathcal{D})$ and $\rho(\mathcal{C})$ covers the same pairs as $\rho(\mathcal{D})$.

Proof. If $\mathcal{C}$ contains no points of degree 1 , there is nothing to prove.

Otherwise let $\tau: P(\mathcal{C}) \rightarrow P(\mathcal{D})$ be an isomorphism from $\mathcal{C}$ to $\mathcal{D}$. Let $\mathcal{A}$ be the set of blocks of $\mathcal{C}$ which contain points of degree 1 . Since $\mathcal{C}$ and $\mathcal{D}$ cover the same pairs, a block containing a point of degree 1 in one of the configurations $\mathcal{C}$ and $\mathcal{D}$ must also occur in the other configuration. Therefore $\mathcal{A} \subseteq \mathcal{C} \cap \mathcal{D}$. Then $\rho(\mathcal{C})=\mathcal{C} \backslash \mathcal{A}$ and $\rho(\mathcal{D})=\mathcal{D} \backslash \mathcal{A}$. Since $\tau(\mathcal{A})=\mathcal{A}$, we have $\rho(\mathcal{C}) \cong \rho(\mathcal{D})$. Furthermore, since we have removed the same pairs from $\mathcal{C}$ and $\mathcal{D}$, the configurations $\rho(\mathcal{C})$ and $\rho(\mathcal{D})$ cover the same pairs.

Lemma 2.2 Suppose $S$ and $S^{\prime}$ are Steiner triple systems and that $n \geq 1$. If there exists a trade $\{\mathcal{C}, \mathcal{D}\}$ with $\mathcal{C} n^{-1}$-isomorphic to $\mathcal{D}$ that transforms $S$ to $S^{\prime}$, then $S$ is $(n+1)^{-1}$-isomorphic to $S^{\prime}$.

Proof. This follows directly from the definitions. 
The converse of Lemma 2.2 is not true. In an attempt to identify the reason for this, we define a pseudo-trade as a pair of configurations $\{\mathcal{C}, \mathcal{D}\}$ such that $\mathcal{C}$ and $\mathcal{D}$ cover the same pairs, $\mathcal{C} \cong \mathcal{D}, \mathcal{C} \cap \mathcal{D} \neq \emptyset$, and for any non-empty subset $\mathcal{A}$ of $\mathcal{C} \cap \mathcal{D}$ we have $\mathcal{C} \backslash \mathcal{A} \neq \mathcal{D} \backslash \mathcal{A}$. By Lemma 2.1, $\mathcal{C}$ and $\mathcal{D}$ have no points of degree 1.

Pseudo-trades of small volume may be enumerated by methods similar to those described in [3]. An example of a pseudo-trade is given by $\{\mathcal{C}, \mathcal{D}\}$ $=\{\{012,034,056,135,146,179,1 b c, 245,37 b, 47 c, 49 b\},\{016,024,035$, $125,13 b, 14 c, 179,347,456,49 b, 7 b c\}\}$, where $\mathcal{C} \cap \mathcal{D}=\{179,49 b\}$. The table below gives, for small volumes, the number of labelled pseudo-trades $\{\mathcal{C}, \mathcal{D}\}$ where configuration $\mathcal{C}$ is canonically labelled [2, page 52].

\begin{tabular}{|c|c|c|c|c|}
\hline$|\mathcal{C}|$ & $\leq 10$ & 11 & 12 & 13 \\
\hline pseudo-trades $\{\mathcal{C}, \mathcal{D}\}$ & 0 & 8 & 24 & 168 \\
\hline
\end{tabular}

With the definition of pseudo-trades in place we have the following result.

Lemma 2.3 Let $S=(V, \mathcal{B})$ and $S^{\prime}=\left(V, \mathcal{B}^{\prime}\right)$ be strictly $2^{-1}$-isomorphic Steiner triple systems. Let $\beta=(|\mathcal{B}|-1) / 2$. Then there exist $T=\{\mathcal{C}, \mathcal{D}\}$ with $\mathcal{C} \cong \mathcal{D}$ and $|\mathcal{C}| \leq \beta$ where $T$ is either a trade or a pseudo-trade and $T$ transforms $S$ to $S^{\prime}$.

Proof. Suppose there exists a $2^{-1}$-isomorphism consisting of a partition of $\mathcal{B}$ into $\mathcal{B}_{0}$ and $\mathcal{B}_{1}$ with $\left|\mathcal{B}_{1}\right| \leq \beta$, a partition of $\mathcal{B}^{\prime}$ into $\mathcal{B}_{0}^{\prime}$ and $\mathcal{B}_{1}^{\prime}$ with $\left|\mathcal{B}_{1}^{\prime}\right| \leq \beta$, and one-to-one mappings $\phi_{0}: V \rightarrow V$ and $\phi_{1}: V \rightarrow V$ such that $\phi_{0}\left(\mathcal{B}_{0}\right)=\mathcal{B}_{0}^{\prime}$ and $\phi_{1}\left(\mathcal{B}_{1}\right)=\mathcal{B}_{1}^{\prime}$. Apply $\phi_{0}^{-1}$ to $S^{\prime}$, let $\mathcal{B}_{1}^{\prime \prime}=\phi_{0}^{-1}\left(\mathcal{B}_{1}^{\prime}\right)$ and consider the pair $\left\{\mathcal{B}_{1}, \mathcal{B}_{1}^{\prime \prime}\right\}$. Note that $\left|\mathcal{B}_{1}\right| \leq \beta$ and that $\left(\mathcal{B} \backslash \mathcal{B}_{1}\right) \cup \mathcal{B}_{1}^{\prime \prime} \cong \mathcal{B}^{\prime}$.

Let $\mathcal{F}=\mathcal{B}_{1} \cap \mathcal{B}_{1}^{\prime \prime}$. If $\mathcal{F}=\emptyset$ then $\left\{\mathcal{B}_{1}, \mathcal{B}_{1}^{\prime \prime}\right\}$ is a trade which satisfies the conditions of the lemma. So we may assume that $\mathcal{F}$ is non-empty. If $\left\{\mathcal{B}_{1}, \mathcal{B}_{1}^{\prime \prime}\right\}$ is a pseudo-trade, we are done. Otherwise there exists a non-empty set $\mathcal{G}$ of maximum cardinality such that $\mathcal{G} \subseteq \mathcal{F}$ and $\mathcal{B}_{1} \backslash \mathcal{G} \cong \mathcal{B}_{1}^{\prime \prime} \backslash \mathcal{G}$. It is clear from the definition that $\left\{\mathcal{B}_{1} \backslash \mathcal{G}, \mathcal{B}_{1}^{\prime \prime} \backslash \mathcal{G}\right\}$ is a trade or a pseudo-trade with the required properties.

The final lemma provides the main ingredient for the proof of Theorem 2.1.

Lemma 2.4 For all admissible $v \geq 27$, there exists an $S T S(v)$ which contains precisely one sub-STS(13). 
Proof. For admissible $v$ such that $27 \leq v \leq 63$, it is straightforward to generate $\operatorname{STS}(v) \mathrm{s}$ with the desired property by Stinson's hill-climbing method [10].

For admissible $v>63$ we employ a recursive construction. Let $G$ be a $\{3\}$ GDD of type $g^{t} h^{u}$ and suppose we have an $\operatorname{STS}(g+13)$ and an $\operatorname{STS}(h+13)$, each having a unique sub-STS(13). Construct a new Steiner triple system, $S$, of order $t g+u h+13$ as follows. Let $T$ be an STS(13). On each group of size $g$, together with the points of $T$, put an $\operatorname{STS}(g+13)$ such that the sub-STS(13) coincides with $T$. Similarly, on each group of size $h$, together with the points of $T$, put an $\operatorname{STS}(h+13)$ such that the sub-STS(13) coincides with $T$.

Suppose, further, that $G$ has at most four groups; i.e. $t+u \leq 4$. We show that the system $S$ has a unique STS(13), namely $T$. To prove this, suppose $U$ is a sub-STS(13) of $S$ and $U \neq T$. Label the groups $G_{1}, G_{2}, \ldots, G_{n}$, where $n=3$ or 4 . Let $A_{i}$ be the set of points of $U$ which lie on $G_{i}$ and let $A$ be the set of points which are common to both $U$ and $T$. Consider three cases according to the size of $A$.

(i) $|A|=0$. For each $i$, we must have $\left|A_{i}\right|=0,1$ or 3 . (We can rule out $\left|A_{i}\right|=7$ and $\left|A_{i}\right|=9$ because we know that neither STS(13) has a sub-STS(7) or a sub-STS(9).) As there are at most four groups, $U$ cannot exist.

(ii) $|A|=1$. Similarly we must have $\left|A_{i}\right|=0$ or 2. Again, there are insufficient groups for $U$ to exist.

(iii) $|A|=3$. Now we are forced to have $\left|A_{i}\right|=0$ for all $i$.

Thus the construction described above preserves the property of containing a unique sub-STS(13). By a theorem of Colbourn, Hoffman and Rees [1], there exist $\{3\}$-GDDs of the following types:

$$
\begin{aligned}
& g^{3} \\
& g^{3} h^{1}, \quad g \equiv h \equiv 0(\bmod 2), h \leq 2 g
\end{aligned}
$$

Using the construction with $\{3\}$-GDDs of these types and starter systems of orders $27,31, \ldots, 63$, we can generate the desired $\operatorname{STS}(v)$ s for all admissible $v>63$ as follows.

First we construct a suitable STS(67) using a $\{3\}$-GDD of type $18^{3}$. Then, using $\{3\}$-GDDs of type $g^{3} h^{1}$ with $g=14$ and $h=14,18,20,24$ and 26, we construct suitable STS $(v)$ s for $v=69,73,75,79$ and 81, respectively. Now let $k \geq 4$ and suppose that we already have suitable STS $(u)$ s for admissible $u$ in the range $27 \leq u \leq 3^{k}$. Let an admissible $v$ be given such that $3^{k}<v \leq 3^{k+1}$, and write $v=6 r+e$, where $e=1$ or 3 . If $r \equiv 0$ or $1(\bmod 3)$, put $s=2 r+1$ 
and $t=36+e$; otherwise put $s=2 r+3$ and $t=30+e$. Let $g=s-13$ and $h=t-13$. Then in either case $2 g-h \geq 4 r-e-47 \geq 0$, since $r \geq 14$ and $e \leq 3$. It is easily verified that $27 \leq s, t \leq 3^{k}$ for admissible $s$ and $t$; hence we can use a $\{3\}$-GDD of type $g^{3} h^{1}$ to construct a suitable Steiner triple system of order $3 g+h+13=v$.

Proof of Theorem 2.1. The two STS(13)s are $2^{-1}$-isomorphic because one can be transformed into the other by a Pasch trade. For the same reason, $\operatorname{STS}(15) \# 1$ is $2^{-1}$-isomorphic to $\operatorname{STS}(15) \# 2$. Pairs of $2^{-1}$-isomorphic $\operatorname{STS}(v)$ s for $v=19,21$ and 25 are easily produced by choosing an appropriate system and transforming it by a Pasch trade.

So let $v \geq 27$ and let $S$ be an $\operatorname{STS}(v)$ which contains a unique subSTS(13), $T$, say. Lemma 2.4 guarantees that $S$ exists. Choose a Pasch configuration in $T$ which when traded transforms $T$ into an STS(13) of the other isomorphism type. Perform this trade thus transforming $S$ into $S^{\prime}$, say. By Lemma 2.2, $S$ is $2^{-1}$-isomorphic to $S^{\prime}$, but clearly $S$ is not isomorphic to $S^{\prime}$.

Proof of Theorem 2.2. Given a positive integer $N$, take $v_{0}(N)$ so large that for all $v \geq v_{0}(N)$, the number of distinct $\operatorname{STS}(v) \mathrm{s}, D(v)$, satisfies

$$
D(v)>(v !)^{N} N^{v(v-1) / 6} .
$$

This is possible because $D(v)=v^{v^{2}(1 / 6+o(1))}$ as $v \rightarrow \infty$ (see [11]). In fact, for large $N, v_{0}(N) \leq N+o(N)$.

Now take any $\operatorname{STS}(v)$, say $S$, with $v \geq v_{0}(N)$. Partition the $v(v-1) / 6$ blocks of $S$ into $N$ sets, some of which may be empty. Such a partition can be represented by a vector of length $v(v-1) / 6$ with entries from 1 to $N$, so that the number of possible partitions is at most $N^{v(v-1) / 6}$.

For each set of the partition, apply a permutation to the base set. The number of combinations of permutations is $(v !)^{N}$. Most resulting sets of triples will not be $\operatorname{STS}(v) \mathrm{s}$ but it is clear that this process can give rise to at most $(v !)^{N} N^{v(v-1) / 6} \operatorname{STS}(v)$ s which are $N^{-1}$-isomorphic to $S$, and any such systems (on the same base set) will arise at least once in this manner. Hence there must exist an $\operatorname{STS}(v)$, say $S^{\prime}$, which is not $N^{-1}$-isomorphic to $S$. But $S^{\prime}$ is certainly $(v(v-1) / 6)^{-1}$-isomorphic to $S$. Hence there exists $n>N$ such that $S$ and $S^{\prime}$ are strictly $n^{-1}$-isomorphic. 
The computational results of this paper suggest that for $v \geq 15$ there exists a pair of $\operatorname{STS}(v) \mathrm{s}$ which are strictly $3^{-1}$-isomorphic.

\section{Algorithms}

Other main results of this paper are two matrices, $D=\left[d_{i, j}\right]$ and $H=\left[h_{i, j}\right]$, showing relations between Steiner triple systems of order 15 . The first is the 'distance table' for STS(15)s, where $d_{i, j}$ is the volume of the smallest trade that transforms $\operatorname{STS}(15) \# i$ into $\operatorname{STS}(15) \# j$, the numbers $i$ and $j$ referring to the standard numbering of the 80 STS(15)s. In the second matrix, $H$, the entry $h_{i, j}$ is the volume of the smallest trade between isomorphic configurations which transforms $\operatorname{STS}(15) \# i$ into $\operatorname{STS}(15) \# j$. We describe two algorithms for computing $\left[d_{i, j}\right]$ and $\left[h_{i, j}\right]$.

\section{Algorithm 3.1}

For $b=4,6,7,8, \ldots$, make a list, $L_{b}$, of all possible trades and pseudotrades $\{\mathcal{C}, \mathcal{D}\}$, where $\mathcal{C}$ is a $b$-block configuration which can occur in an $\operatorname{STS}(15)$.

For each $\operatorname{STS}(15), S$, for each $\{\mathcal{C}, \mathcal{D}\} \in L_{b}$ :

For each occurrence $\phi(\mathcal{C})$ of an isomorphic copy of $\mathcal{C}$ in $S$ : transform $S$ to $S^{\prime}$, say, by the trade or pseudo-trade $\{\phi(\mathcal{C}), \phi(\mathcal{D})\}$. Record the designation $(01-80)$ of $S$ and $S^{\prime}$ as well as information about the trade.

\section{Algorithm 3.2}

For $b=4,6,7,8, \ldots$, for each $\operatorname{STS}(15), S$, for each set $\mathcal{C}$ of $b$ blocks of $S$ :

For each trade or pseudo-trade $\{\mathcal{C}, \mathcal{D}\}$ : record the designation of $S$ and $S^{\prime}$, the $\operatorname{STS}(15)$ that results from transforming $S$ by $\{\mathcal{C}, \mathcal{D}\}$, as well as information about $\{\mathcal{C}, \mathcal{D}\}$.

In spite of its apparent naivety, Algorithm 3.2 is the preferred option. It turns out that Algorithm 3.1 is not practicable for dealing with $b \geq 10$ because of the difficulty of constructing the list $L_{b}$. On the other hand, 
Algorithm 3.2 does not require a predetermined list and, furthermore, there is an efficient method, described in [3], for constructing all possible trades $\{\mathcal{C}, \mathcal{D}\}$, if any, from a given configuration $\mathcal{C}$. Also it is clear from [3] how to adapt the procedure to construct pseudo-trades. In fact, we used both methods for $b \leq 9$ and thereby gave ourselves extra confidence that our computer programming was sound.

There are a number of ways to shorten the computational effort and reduce the amount of work to a reasonable level. We mention three observations. (i) A configuration that is part of a trade or a pseudo-trade has no points of degree one. (ii) To prove that two STS(15)s are $2^{-1}$-isomorphic, we do not need to consider trades or pseudo-trades of volume greater than 17. This follows from the proof of Lemma 2.3. (iii) In computing the matrix $H$, after examining all trades of volume less than or equal to 17 , a complete list of pairs $(i, j)$ where $h_{i, j}>17$ is known. If in addition we know that the smallest pseudo-trade which transforms $\operatorname{STS}(15) \# i$ to $\operatorname{STS}(15) \# j$ has volume $p \leq 17$, we can deduce that either $h_{i, j} \leq 35-p$ or $h_{i, j}=\infty$, thus limiting the search space.

\section{Results}

The two matrices $D$ and $H$ are presented in tabular form. For clarity, only the upper half of the matrix is given; the other half follows by symmetry.

In Table 4.1 , the entry $(i, j), i \leq j$, indicates $d_{i, j}$, the volume of the smallest trade that transforms $\operatorname{STS}(15) \# i$ to $\operatorname{STS}(15) \# j$. We do not distinguish between trades with isomorphic configurations and trades with non-isomorphic configurations. Numbers 10, 11, .., 19 are represented by lower-case letters $a, b, \ldots, j$, respectively. We find that any STS(15) can be transformed into any other STS(15) by a trade of at most 19 blocks. Also 19 blocks are necessary only for the pairs $\{\operatorname{STS}(15) \# 01, \operatorname{STS}(15) \# 62\}$ and $\{\operatorname{STS}(15) \# 01, \operatorname{STS}(15) \# 71\}$. Eighteen blocks suffice for the rest. If STS(15) \#01 is excluded, then 17 blocks are sufficient, and sometimes necessary.

Table 4.2 has the same format as Table 4.1 except that each trade consists of a pair of isomorphic configurations. The entry $(i, j), i \leq j$, indicates $h_{i, j}$, the volume of the smallest such trade that transforms $\operatorname{STS}(15) \# i$ to STS(15) \#j. A dot indicates that no such trade exists: $h_{i, j}=\infty$. The same scheme as above is used for representing two-digit numbers, and entries that 
differ from the corresponding values in Table 4.1 are underlined. (Observe that values 4, 6, 7 and 8 occur at precisely the same locations in both tables.) There are only two values greater than $17: h_{06,31}=20$, represented by the letter $\mathrm{k}$ in the table, and $h_{07,25}=24$, represented by the letter 0 .

Let $\nu$ be the smallest $n$ such that every pair of STS(15)s is $n^{-1}$-isomorphic. Lemma 2.3 and the existence of pairs $(i, j)$ where $h_{i, j}=\infty$ and $d_{i, j}>17$, at $(01,71)$ for example, implies that $\nu \geq 3$. However, from the information in Table 4.2 it is easy to deduce that $\nu \leq 4$. The table shows that STS(15) \#11 is $2^{-1}$-isomorphic to every other STS(15) except possibly STS(15) \#01. Therefore it follows from Proposition 6 of [8] that for $2 \leq i<j \leq 80, \operatorname{STS}(15) \# i$ is $4^{-1}$-isomorphic to $\operatorname{STS}(15) \# j$. In a similar manner we can show that $\operatorname{STS}(15) \# 01$ is $4^{-1}$-isomorphic to $\operatorname{STS}(15) \# j$ for $2 \leq j \leq 80$ by identifying a system $\operatorname{STS}(15) \# k$ which is $2^{-1}$-isomorphic to both $\operatorname{STS}(15) \# 01$ and $\operatorname{STS}(15) \# j$.

Table 4.2 shows that all except 537 pairs of STS(15)s are $2^{-1}$-isomorphic. However, to ascertain the full extent of $2^{-1}$-isomorphism we must also determine $2^{-1}$-isomorphic pairs of STS(15)s which are not indicated by Table 4.2. Their existence is possible because the converse of Lemma 2.2 is false. It suffices, by Lemma 2.3, to look for pseudo-trades of volume not greater than 17. In fact, for given $i, j$, all we need to do is allow common blocks in the search for $\mathcal{C}, \mathcal{D}$ with the smallest $|\mathcal{C}|$ such that $\mathcal{C}$ and $\mathcal{D}$ cover the same pairs, $\mathcal{C} \cong \mathcal{D}$ and $\{\mathcal{C}, \mathcal{D}\}$ transforms $\operatorname{STS}(15) \# i$ to $\operatorname{STS}(15) \# j$. If $\mathcal{C} \cap \mathcal{D}$ is empty, $\{\mathcal{C}, \mathcal{D}\}$ is a trade; otherwise it is clear that $\{\mathcal{C}, \mathcal{D}\}$ is a pseudo-trade. Moreover, we can assume that $|\mathcal{C} \backslash \mathcal{D}| \geq 9$; for otherwise $\{\mathcal{C} \backslash \mathcal{D}, \mathcal{D} \backslash \mathcal{C}\}$ is a trade of volume at most 8 and therefore $\{\mathcal{C} \backslash \mathcal{D} \cong \mathcal{D} \backslash \mathcal{C}\}$. A complete search produces a further six $2^{-1}$-isomorphic pairs. Specifically, let $e_{i, j}$ denote the smallest volume of a pseudo-trade, if any, that transforms $\operatorname{STS}(15) \# i$ to $\operatorname{STS}(15) \# j$. Then we have the following values for pairs $(i, j)$ where $i<j$ and $h_{i, j}=\infty$.

\begin{tabular}{|c|c|c|c|c|c|c|}
\hline$i, j$ & 05,30 & 05,34 & 12,71 & 16,29 & 19,67 & 19,72 \\
\hline$e_{i, j}$ & 15 & 15 & 15 & 15 & 17 & 16 \\
\hline
\end{tabular}

It is also worth mentioning that in the only two cases where $17<h_{i, j}<\infty$ we have $e_{06,31}=e_{07,25}=11$.

Thus $2^{-1}$-isomorphism accounts for all except $537-6=531$ pairs of STS(15)s. To establish a $3^{-1}$-isomorphism for the remaining pairs, three approaches may be used. Let $S$ and $S^{\prime}$ be $\operatorname{STS}(15)$ s which are not $2^{-1}$ isomorphic. (i) As in the proof of Proposition 6 of [8], it is sufficient to find an $\operatorname{STS}(15), S^{\prime \prime}$, and trades, $\{\mathcal{C}, \mathcal{D}\}$ and $\{\mathcal{E}, \mathcal{F}\}$, where $\mathcal{C} \cong \mathcal{D}$ and $\mathcal{E} \cong \mathcal{F}$, 
such that $\{\mathcal{C}, \mathcal{D}\}$ transforms $S^{\prime \prime}$ to $S,\{\mathcal{E}, \mathcal{F}\}$ transforms $S^{\prime \prime}$ to $S^{\prime}$ and either $\mathcal{C} \cap \mathcal{E}=\emptyset$, or $\mathcal{C} \subseteq \mathcal{E}$, or $\mathcal{E} \subseteq \mathcal{C}$. (ii) We find a trade that consists of $2^{-1}$ isomorphic configurations, possibly the one which was used to establish the value of the corresponding entry in Table 4.1, and then apply Lemma 2.2. (iii) We find a trade, $\{\mathcal{C}, \mathcal{D}\}$ that transforms $S$ to $S^{\prime}$ and a set of blocks $\mathcal{X}$ of $S$ disjoint from $\mathcal{C}$ such that $\mathcal{C} \cup \mathcal{X}$ is $2^{-1}$-isomorphic to $\mathcal{D} \cup \mathcal{X}$.

The second approach is particularly effective. Elementary computation shows that every trade of volume at most 12 consists of a pair of $2^{-1}$ isomorphic configurations. This accounts for every pair where there is a value of c or less in Table 4.1. Further, with a little more computation we can use the same method to establish the required $2^{-1}$-isomorphism for the trades corresponding to entries in Table 4.1 with values $d, e, f$ and $g$. Hence for pairs $\{i, j\}$ where there is one of these letters in Table 4.1 and a dot in Table 4.2, we have that $\operatorname{STS}(15) \# i$ is $3^{-1}$-isomorphic to $\operatorname{STS}(15) \# j$.

Of the remaining 39 cases, where the value in Table 4.1 is $h$, $i$ or $j$, $3^{-1}$-isomorphic pairs have been found; four by method (i): $(01, j), j=$ $33,64,76,79$; a further 33 pairs by method (ii): $(01, j), j=36,37,38$, $41,44,45,46,48,49,50,52,53,55,56,57,58,60,61,63,65,66,67,68,69$, $70,71,72,74,75,77$, as well as $(02,77),(03,80)$ and $(16,80)$; and two pairs by method (iii): $(01,43)$ and $(01,62)$.

Thus we have proved the following.

Theorem 4.1 Any two Steiner triple systems of order 15 are $3^{-1}$-isomorphic.

Two particular cases of the final 39 , namely $(01,43)$ and $(01,62)$, required considerable amounts of computer time, mainly because methods (i) and (ii) failed to produce the desired results. So it is appropriate to give details of these $3^{-1}$-isomorphisms. 
In the first case we have:

STS(15) \#43: 012034057 06a 08c 09d 0be 135146 17c 189 1ae 1bd 236247258 29a 2bc 2de 37d 38b 39e 3ac 459 $48 \mathrm{e} 4 \mathrm{ab} 4 \mathrm{~cd} 56 \mathrm{~b} 5 \mathrm{ad} 5 \mathrm{ce} 67 \mathrm{e} 68 \mathrm{~d}$ 69c 78a 79b;

extended trade: (06a 08c 09d 135 17c 189 1ae 247 29a 39e $3 a c 459$ 4cd 5ad 5ce 68d 69c 78a 146, 069 08a 0cd 139 15a 178 1ce 249 27a 35c 3ae 45d 47c 59e 68c 6ad 89d 9ac 146) $=(\mathcal{C}, \mathcal{D})$;

$\mathcal{C}_{1}: \quad \quad$ 08c 09d 29a 39e 4cd 5ad 68d 69c 78a,

$\mathcal{D}_{1}: \quad 06908 \mathrm{a} 13915 \mathrm{a} 178249$ 68c 6ad 89d,

$\mathcal{C}_{2}$ : $\quad$ 06a 13517 c 189 1ae 247 3ac 459 5ce 146,

$\mathcal{D}_{2}$ : $\quad$ 0cd 1ce 27a 35c 3ae 45d 47c 59e 9ac 146.

In the second case we have:

STS(15) \#62: 012034057068 09b 0ad 0ce 135146 17a 18b 19e 1cd 236245 27b 28c 29d 2ae 37c 38d 39a 3be 47d 48 e 49c 4ab 569 58a 5bc 5de 67e 6ac 6bd 789;

extended trade: (012 034 09b 0ad 0ce 17a 19e 1cd 29d 37c 39 a 3be 49c 4ab 5bc 5de 67e 6ac 6bd 245, 01e 02d 03c 04b 09a 129 17c 1ad 349 37e

$3 \mathrm{ab} 4 \mathrm{ac} 5 \mathrm{bd} 5$ ce 67 a 6 be $6 \mathrm{~cd} 9 \mathrm{bc} 9 \mathrm{de} 245)=(\mathcal{C}, \mathcal{D})$;

$\mathcal{C}_{1}: \quad \quad 09 \mathrm{~b} 19 \mathrm{e} 1 \mathrm{~cd} 29 \mathrm{~d}$ 3be 49c 5bc 67e 6ac 6bd,

$\mathcal{D}_{1}$ : $\quad$ 01e 02d 03c 09a 1ad 4ac 5ce 67a 6be 6cd,

$\mathcal{C}_{2}: \quad 012034$ 0ad 0ce 17a 37c 39a 4ab 5de 245,

$\mathcal{D}_{2}: \quad \quad$ 04b $12917 \mathrm{c} 34937 \mathrm{e} 3 \mathrm{ab} 5 \mathrm{bd} 9 \mathrm{bc} 9 \mathrm{de} 245$.

In each case one can verify that the extended trade transforms the given STS(15) into $\operatorname{STS}(15) \# 01$. 


\section{References}

[1] C. J. Colbourn, D. G. Hoffman and R. S. Rees, A new class of group divisible designs with block size three, J. Combin. Theory Ser. A 59 (1992), 73-89.

[2] C. J. Colbourn and A. Rosa, Triple Systems, Oxford University Press, New York 1999.

[3] A. D. Forbes, M. J. Grannell and T. S. Griggs, Configurations and trades in Steiner triple systems, Australas. J. Combin. 29 (2004), 75-84.

[4] P. Kaski and P. R. J. Östergård, The Steiner triple systems of order 19, Math. Comp. 73, no.248 (2004), 2075-2092.

[5] T. P. Kirkman, On a problem in combinatorics, Cambridge and Dublin Math. J. 2 (1847), 191-204.

[6] C. C. Lindner and A. Rosa, Steiner triple systems having a prescribed number of triples in common, Canad. J. Math. 27 (1975), 1166-1175. Corrigendum: 30 (1978), 896.

[7] E. H. Moore, Concerning triple systems, Math. Ann. 43 (1893), 271-285.

[8] G. Quattrocchi and G. Rinaldi, Steiner systems and $n^{-1}$-isomorphisms, J. Geom. 58 (1997), 146-157.

[9] M. V. Ramana, E. R. Scheinermann and D. Ullman, Fractional isomorphism of graphs, Discrete Math. 132 (1994), 247-265.

[10] D. R. Stinson, Hill-climbing algorithms for the construction of combinatorial designs, Ann. Discrete Math. 26 (1995), 311-334.

[11] R. M. Wilson, Nonisomorphic Steiner triple systems, Math. Z. 135 (1974), 303-313. 
Table 4.1: $d_{i, j}$, the distance between STS(15)s, part I

\begin{tabular}{|c|c|c|}
\hline & $\begin{array}{l}0000000001111111111222222222233333333334 \\
1234567890123456789012345678901234567890\end{array}$ & $\begin{array}{l}4444444445555555555666666666677777777778 \\
1234567890123456789012345678901234567890\end{array}$ \\
\hline 01 & 04688ccaccdccae7deefffgeedffgggghggiihgg & hgiiiigiiighhghhihghhjiiiihiiijhgiiiigif \\
\hline 02 & 044488688a886a6aacccccccacccdcdedefgfdd & eeggffefffeeedeefedfefefffefffgfeffehegf \\
\hline 03 & 04486487a7648478cbbbba98aabcbcdddefddc & ceeeeedeeecdccddedddefeeefeef ef eeefegefh \\
\hline 04 & 044844474446886a8998886998a8acbccedba & bcedcdccddcccbcccbadcccdcdcddcddecdcecef \\
\hline 05 & 04446477468868a9bb8988aaab8bcccdedcc & ccddcdcdddcdcccddcbdcddddecddcedccddf cef \\
\hline 06 & 0486647774 c8488999999889898aabcccbb & bccccebccccebbccebccacacccdccccddccddddf \\
\hline 07 & 08977b678a48abbcac9abbbaaaccdecdcd & dcccecceddcfeddedddccfdddededdeecedfdccf \\
\hline 08 & 0448744444488bb776477797aa9abdba9 & 9 cbbbcbbcbaba9abbbabcbaccccccbcccbcaecdf \\
\hline 09 & $0447444874849944466647778899 \mathrm{cb} 99$ & 9bba9b9ababa9a9aa97a99aaabbbb9bcdaa8ccde \\
\hline 10 & 04444487477994644777747989 acaa9 & 9baa9b9bbbaba99ab99aaaabababbabbb9b9dabe \\
\hline 11 & $04774 \mathrm{c} 77446666774474747798 \mathrm{~b} 988$ & 8a889b89999a98aa988a897aa9aaa8aab989aaac \\
\hline 12 & $0777 a b 86466444488476788 a a d b 88$ & 8bba9b9abbaa999bb87a9a9aababbabbdaa8dcde \\
\hline 13 & 04484477bb7747886879989beab9 & 9aaabbabbbabaa9cbaaabbacbccbcccccbbbdddd \\
\hline 14 & 07667b7bb7764888989a9aaeba9 & 9 cccbcbbcbaca9babaabbccccccccadcecdbdcef \\
\hline 15 & 084447997777477877 aab8aaab & 9aaabcaab9baaaab99aaba7ababbaabbb9aadcce \\
\hline 16 & 068ccddaa97bbbcbcdbcdfddb & cedededeeecdcbddeeddfedeeefeeefefefdgffh \\
\hline 17 & 0477bb9a77779aaa99bacbbb & baaabacbbbaccbbcacaaccacccbdbacccbcddccd \\
\hline 18 & 074a97777747a7977bbb99a & bcba9ba9ab9aa9aaab8a9a9ababbb8abcabbdaef \\
\hline 19 & 0668999977a79799aacb99 & 9baaab8bb9bbaaccbaaa9a4acbbbbabbababcaac \\
\hline 20 & 086777777779744 aac9aa & abba99a999989899a84869989bb998aabaaaabce \\
\hline 21 & $0498889888 a 8 a a 499844$ & 4a998848889a77a9a7ab67899aa9a99aa8a9bbbd \\
\hline 22 & 0899989a9a8888bb977 & 79baaa7a99884488848944a79999aa9ababbbadd \\
\hline 23 & $04444477447497 b 988$ & 88a76868899988a89687b87a8888978ab877aabd \\
\hline 24 & $04444764444 a 7 a 968$ & $88878868889768989877 b 8889888979 a b 874 a 9 b c$ \\
\hline 25 & $044444766468 \mathrm{~b} 886$ & 6a779889888888698877b9799a9a98a9ba97baad \\
\hline 26 & $04447477787 b 986$ & 6a998a88a88986888889b9899aaa97a9b997babe \\
\hline 27 & $0474747794 \mathrm{~b} 888$ & 68779a88869888896679a948998887988677aaac \\
\hline 28 & 0777744878668 & 8aa96886886786889947a9778998a478a897b8cc \\
\hline 29 & 0874478 aca88 & 6ba788999897899a8969a668a9a9a8abbb7aaacd \\
\hline 30 & $0444684 b 886$ & 68448888999868889879aa77888a8798b879977d \\
\hline 31 & 0777 a8a899 & 9b778b8baaba9999a9a9caaaa8bab99a8697c88c \\
\hline 32 & $06487 a 968$ & $68848868688766889847 a 64886899478884877 a c$ \\
\hline 33 & $0487 a 888$ & $887866886674768786778994888884888897 a 99 b$ \\
\hline 34 & $089 a 688$ & $889786868867668686749977688864888888 a 8 b c$ \\
\hline 35 & $08 b 474$ & 4b86787798774898a899a6669a977899a7bb98bd \\
\hline 36 & 08896 & 8a44888989879a9999abcb7a98b8a99bc8aab88c \\
\hline 37 & Obab & 94ba886988ab98998ac9cbaa977abaa9899bbb9c \\
\hline 38 & 047 & $78884777746668648888 b 99877744688 a 98 a 968 c$ \\
\hline 39 & 04 & $7998448444687776848898 \mathrm{a} 967977687778698 \mathrm{bb}$ \\
\hline 40 & 0 & $49684774778877777488999887969989 a 488 b 98 b$ \\
\hline
\end{tabular}


Table 4.1: $d_{i, j}$, the distance between STS(15)s, part II

\begin{tabular}{|r|r|}
\hline & 4444444445555555555666666666677777777778 \\
& 1234567890123456789012345678901234567890 \\
\hline 41 & $09884747747874767788988878888868 \mathrm{a} 79899 \mathrm{ab}$ \\
42 & $0886686678896688886 \mathrm{~b} 9 \mathrm{~b} 9644888964 \mathrm{a} 6 \mathrm{~b} 88 \mathrm{~b} 9$ \\
43 & $04898898979 \mathrm{aa} 989 \mathrm{abcba} 988 \mathrm{a} 8 \mathrm{ba} 9 \mathrm{bb} 8 \mathrm{abb} 74 \mathrm{c}$ \\
44 & $0688689747987998 \mathrm{ac} 87988899879 \mathrm{a} 979947 \mathrm{c}$ \\
45 & $0747646687477788 \mathrm{~b} 798448878678869888 \mathrm{c}$ \\
46 & $044488887686766 \mathrm{a} 9887466866647889 \mathrm{ab9}$ \\
47 & $0447697478878898687886889774898 \mathrm{a} 9 \mathrm{~b}$ \\
48 & $0477887667788 \mathrm{~b} 98847686688879 \mathrm{a} 99 \mathrm{bb}$ \\
49 & $077686774888 \mathrm{aa} 984676866477688 \mathrm{ab} 8$ \\
50 & $068877766 \mathrm{a} 8 \mathrm{aa} 6874677686888966 \mathrm{ab}$ \\
51 & $0444444876 \mathrm{~b} 797466767789899 \mathrm{a} 4 \mathrm{bc}$ \\
52 & $047647747 \mathrm{c} 7987888644688 \mathrm{a} 7 \mathrm{a} 8 \mathrm{ab}$ \\
53 & $04747488848478868777 \mathrm{a} 99887 \mathrm{bd}$ \\
54 & $0464466778766867888878 \mathrm{a} 97 \mathrm{ac}$ \\
55 & $074766 \mathrm{a} 4 \mathrm{~b} 987664666886888 \mathrm{bc}$ \\
56 & $07477 \mathrm{~b} 7 \mathrm{a} 767846644 \mathrm{aa} 9887 \mathrm{bc}$ \\
57 & $0486978666687887688 \mathrm{a} 87 \mathrm{ba}$ \\
58 & $098778798679977978998 \mathrm{bc}$ \\
59 & $04 \mathrm{a} 966688687888 \mathrm{a} 8 \mathrm{babcc}$ \\
60 & $0 \mathrm{ba} 9966676446694778 \mathrm{ba}$ \\
61 & $07 \mathrm{a} 4 \mathrm{~b} 9 \mathrm{baaabbccdddddf}$ \\
62 & $09689997869 \mathrm{ba} 898 \mathrm{bcd}$ \\
63 & $09 \mathrm{a} 9888798 \mathrm{~b} 97 \mathrm{a} 9 \mathrm{aca}$ \\
64 & $08688878 \mathrm{a} 99 \mathrm{aabaac}$ \\
65 & $06464664898997 \mathrm{aa}$ \\
66 & $04468776 \mathrm{a} 89889 \mathrm{a}$ \\
67 & $0466746 \mathrm{a} 8969 \mathrm{cb}$ \\
68 & $04747898999 \mathrm{cb}$ \\
69 & $08649 \mathrm{a} 8 \mathrm{a} 68 \mathrm{bc}$ \\
70 & $0488884 \mathrm{a} 6 \mathrm{ba}$ \\
71 & $068 \mathrm{a} 7788 \mathrm{ca}$ \\
72 & $08 \mathrm{a} 6 \mathrm{~b} 68 \mathrm{c} 9$ \\
73 & $0 \mathrm{a} 9 \mathrm{ba} 886$ \\
74 & 0898668 \\
75 & $0 \mathrm{a} 4 \mathrm{aac}$ \\
76 & $0 \mathrm{~b} 9 \mathrm{dc}$ \\
77 & $0 \mathrm{bbb}$ \\
78 & $08 \mathrm{~b}$ \\
79 & $0 \mathrm{c}$ \\
80 & 0 \\
\hline & \\
\hline &
\end{tabular}


Table 4.2: $h_{i, j}$ for pairs of STS(15)s, part I

\begin{tabular}{|c|c|c|}
\hline & $\begin{array}{l}0000000001111111111222222222233333333334 \\
1234567890123456789012345678901234567890\end{array}$ & $\begin{array}{l}4444444445555555555666666666677777777778 \\
1234567890123456789012345678901234567890\end{array}$ \\
\hline 01 & $04688 . c a \cdot \underline{e} . c \ldots 7 \ldots \ldots \ldots \ldots$....... & $\ldots \ldots \ldots f$ \\
\hline 02 & $044488688 \underline{c} 886 a 6 a$. с. .сcса. .cee. . . . . . . & $\ldots \ldots \ldots \ldots \ldots \ldots \ldots \ldots$ eh $\ldots \ldots$ \\
\hline 03 & $04486487 a 7648478 \mathrm{cbcccca} 8 \underline{c} \cdot \mathrm{bcce} . . . \ldots$ & 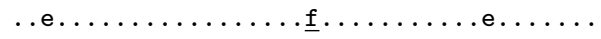 \\
\hline 04 & 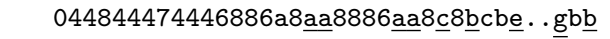 & $\underline{\mathrm{d}} \ldots \underline{\operatorname{ded}} \ldots . \underline{\operatorname{dc}} \underline{\mathrm{df} h} . \underline{\mathrm{fcdc}} \ldots \underline{\mathrm{e}} \ldots \underline{\mathrm{d}} \ldots \ldots \underline{\mathrm{f}} . \mathrm{c} \ldots$ \\
\hline 05 & 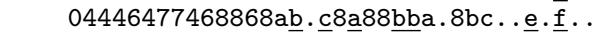 & 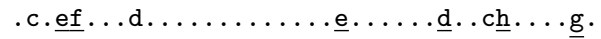 \\
\hline 06 & $0486647774 \mathrm{c} 8488 \underline{\mathrm{acbbbb}} 88 \underline{\mathrm{c}} 8 \underline{\mathrm{k}} 8 \underline{\mathrm{cc}} \ldots . \mathrm{.bb}$ & 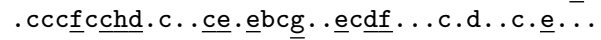 \\
\hline 07 & 08a77.678.48a.ff..o...b.......... & $\ldots$ f................eccf \\
\hline 08 & 0448744444488 . .7764777aㅡab․ & $\underline{b} . \underline{c c c c b d d} . \underline{c b c a c b} . \underline{c} a b \ldots \underline{c} c \underline{d} \ldots$. . bd $\ldots \underline{f e c} \ldots$ \\
\hline 09 & $044744487484 \mathrm{aa} 4446664 \overline{7} 77 \overline{88} \mathrm{~b} \bar{b} \cdot \overline{d a} \cdot \bar{a}$ & ac. bbbabbbbaaadada 7 baaaacddbcbdd. bb8cf. . \\
\hline 10 & $04444487477 \underline{\overline{\mathrm{aa}}} 4644777747 \underline{\mathrm{a}} 8 \overline{\mathrm{ab}} \cdot \overline{\mathrm{bbb}}$ & 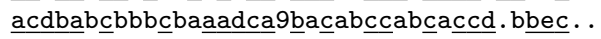 \\
\hline 11 & $04774 c 7744 \overline{66} 66774474747 \overline{7} \bar{a} 8 \underline{e} \bar{a} 88$ & 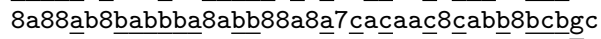 \\
\hline 12 & $0777 \underline{c b} 86466444488476788 \mathrm{a} \underline{e} \cdot \underline{\mathrm{c}} 88$ & 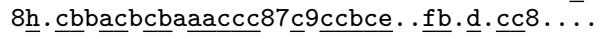 \\
\hline 13 & $04484477 \underline{c c} 774788687998 \underline{e} \bar{b} \cdot \underline{\bar{b}} \cdot \underline{a}$ & 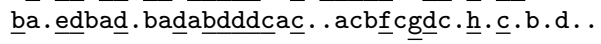 \\
\hline 14 & $07667.7 \underline{\mathrm{cf}} 7764888989 \underline{\mathrm{bab}} \ldots . . \mathrm{e}$ & 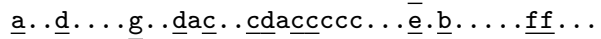 \\
\hline 15 & 084447ㅁaa7777477877aab8abab & 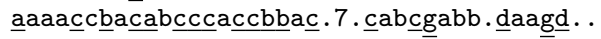 \\
\hline 16 & 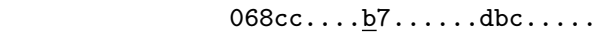 & 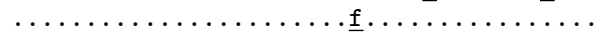 \\
\hline 17 & $0477 \underline{f c} 9 a \overline{7} 777$ abea99.e $c \underline{d} .$. & 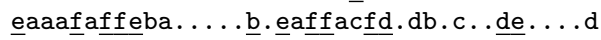 \\
\hline 18 & 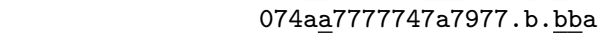 & 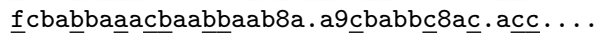 \\
\hline 19 & $0668 \underline{\mathrm{ba}} 9977 \underline{\mathrm{b}} 797 \underline{\mathrm{bcc}} . . \overline{\mathrm{b}} \cdot \underline{\mathrm{b}}$ & 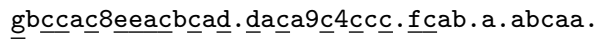 \\
\hline 20 & $08677777777 \underline{\text { a } 744 a a c b a a ~}$ & 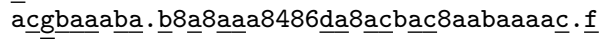 \\
\hline 21 & 04a888a888ㅁ8aa4a .844 & 4므. \\
\hline 22 & $0 \overline{8}$ aaa 8 acaé8888 & $7 \bar{b}$ db $a a 7$ baa $884488848 \underline{a} 44 a 7$ bbaabaaacde. \\
\hline 23 & 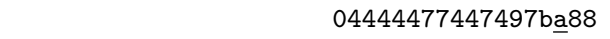 & $8 \overline{8 a} 768688 \underline{a} 9988 a 8 \underline{a} 687.87 \underline{b} 8888978 \mathrm{ab877} \overline{\mathrm{aa}}$. \\
\hline 24 & $04444764444 a 7 a$ & $8887886888 \underline{a} 768989877 \mathrm{~b} 888 \underline{a} 888 \underline{b} 79 \mathrm{ab} 874 a 9 \underline{c}$. \\
\hline 25 & $044444766468 \underline{d} 886$ & 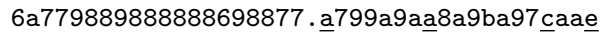 \\
\hline 26 & 04447477787.986 & 6a998a88a88986888889. a899ㅁaa97a9ca $97 \underline{c c}$. \\
\hline 27 & $04747477 \underline{a} 4 \underline{c} 888$ & $68779 \mathrm{a} 88869888896679 \underline{\underline{9}} \overline{9} 489 \overline{9} 8887988677 \overline{a a a f}$ \\
\hline 28 & 0777744878668 & 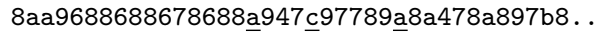 \\
\hline 29 & 0874478 aca88 & $6 \underline{c c} 788 \underline{a} 998978 \underline{a a a} 8 \underline{a} 69 \underline{c} 668 a \underline{b} a 9$ b $8 a b \underline{d} .7 a a a \cdot g$ \\
\hline 30 & $0444684 \underline{e} 886$ & $684488889 \underline{a} 9868889879 \underline{\mathrm{cb}} 77888 \mathrm{a} 8798 \mathrm{~b} 879977 \overline{\mathrm{d}}$ \\
\hline 31 & $0777 \underline{c 8 a} 8 \underline{a a}$ & ab778b8cbabaaa99baa9. caaç 8 cbb99a 8697 e-88e \\
\hline 32 & $06487 a 968$ & $6884886868876688 \underline{a} 847 \underline{c} 6488689 \underline{a} 478884877 \underline{c d}$ \\
\hline 33 & $0487 \underline{b} 888$ & $8878668866747687 \overline{8} 677 \overline{8} \underline{a} 94888884888897 \mathrm{ab} \overline{9 b}$ \\
\hline 34 & $089 \underline{b} 688$ & $88 \underline{a} 78686886766868674 \underline{e} \overline{9} 77688864888888 a \overline{8} \underline{c}$. \\
\hline 35 & $08 \underline{e} 474$ & 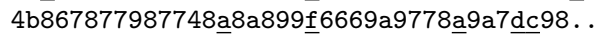 \\
\hline 36 & $088 \underline{a} 6$ & $8 a 4488898 \underline{a} 879 a 9999 a \underline{c} c b 7 a 98 b 8 a 99 b c 8 a a b 88 c$ \\
\hline 37 & Obab & 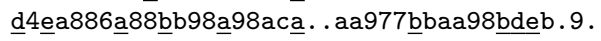 \\
\hline 38 & 047 & 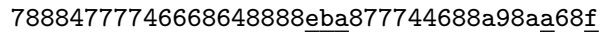 \\
\hline 39 & 04 & 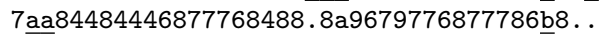 \\
\hline 40 & 0 & $4 \underline{a} 684774778877777488 \underline{a a a} 887 \underline{a} 69989 a 488 b \underline{a}$ - 8 . \\
\hline
\end{tabular}


Table 4.2: $h_{i, j}$ for pairs of STS(15)s, part II

\begin{tabular}{|c|c|}
\hline & $\begin{array}{l}44444444455555555556666666666777777777778 \\
1234567890123456789012345678901234567890\end{array}$ \\
\hline 41 & Ob884747747874767788c88878888868a7989a.b \\
\hline 42 & $0886686678896688886 . c b 9644888964 d 6 \mathrm{~d} 88 \overline{c c}$ \\
\hline 43 & 0489889897aaa98aab.fa988b8ba9be8ccc74f \\
\hline 44 & 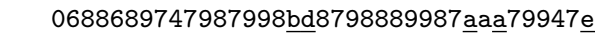 \\
\hline 45 & $0747646687477788 \underline{c} 7 \underline{a} 8448878678869888$. \\
\hline 46 & 044488887686766a9887466866647889acb \\
\hline 47 & 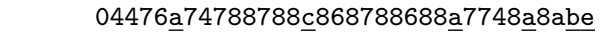 \\
\hline 48 & 0477887667788d्d98847686688879a99. . \\
\hline 49 & 077686774888aaa84676866477688ad8 \\
\hline 50 & $068877766 \mathrm{a} 8 . \mathrm{a} 6874677686888 \underline{b} 66 \mathrm{af}$ \\
\hline 51 & 0444444876b7974667677898a9a4d. \\
\hline 52 & 047647747 $\underline{\mathrm{d}} 7 \underline{\mathrm{a}} \mathrm{8} 7888644688 \mathrm{a} 7 \mathrm{a} 8 \mathrm{ab}$ \\
\hline 53 & 04747488848478868777a9a887bd \\
\hline 54 & $0464466778766867888878 a 97 a$. \\
\hline 55 & 074766므4b987664666886888ㅁ. \\
\hline 56 & 07477무7a767846644ㅁa9887. . \\
\hline 57 & $0486 \underline{e} 78666687887688 \mathrm{a} 87 \underline{c} a$ \\
\hline 58 & 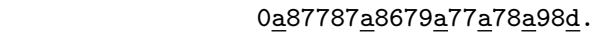 \\
\hline 59 & 04되의 \\
\hline 60 & Obaą966676446694778ㅁa \\
\hline 61 & $07.4 \underline{d c b a a d} . b \underline{d} . . . d .$. \\
\hline 62 & 09689997869da898b. . \\
\hline 63 & 09ba888798ca7a9aca \\
\hline 64 & 08688878a99acbaae \\
\hline 65 & 06464664898 ba7d. \\
\hline 66 & $04468776 a 89889 a$ \\
\hline 67 & $0466746 a 8 \underline{a} 69 \mathrm{cb}$ \\
\hline 68 & 047478a8999ce \\
\hline 69 & $08649 a 8 a 68 \underline{c} c$ \\
\hline 70 & 0488884a6ea \\
\hline 71 & 068a7788ca \\
\hline 72 & $08 \mathrm{a} 6 \mathrm{~b} 68 \mathrm{~d} 9$ \\
\hline 73 & 0aada886 \\
\hline 74 & 0898668 \\
\hline 75 & 0a4aa. \\
\hline 76 & $\mathrm{Ob}_{\mathrm{c}} .$. \\
\hline 77 & $\mathrm{ob}$. \\
\hline 78 & $08 \mathrm{~b}$ \\
\hline 79 & 0 . \\
\hline 80 & 0 \\
\hline
\end{tabular}

